\title{
Schmerztherapie 2005 - Ein schmerzhafter Spagat?
}

$\mathrm{D}$ ie Schmerztherapie wurde in den vergangenen Jahrzehnten zu Recht häufig als ein Stiefkind der Medizin bezeichnet. In der Tat war die Diskussion über die Schmerztherapie fokussiert auf ihre Gefahren wie Sucht, Atemdepression und Gewöhnung. Deutsche Medizinstudenten lernten (wenn überhaupt) nur in pharmakologischen Vorlesungen einiges über Analgetika. Und Schmerzen, die sich morphologisch, und das hieß fast immer radiologisch oder neurografisch nicht erklären ließen, galten als „Beschwerden ohne Korrelat“, - eine vornehme Umschreibung für „psychogen“, weshalb diese Patienten in erster Linie mit „schmerzdistanzierenden" Medikamenten wie Neuroleptika oder Tranquilizer behandelt oder, besser gesagt, ruhig gestellt wurden.

Hieran hat sich ohne Zweifel vieles gebessert. Inzwischen ist die Spezielle Schmerztherapie eine eigene Zusatzbezeichnung geworden, manche wünschen hierfür sogar einen neuen Facharzt. Heute ist ein Onkologe, der keine qualifizierte Schmerztherapie mit Opioiden betreibt, glücklicherweise selten geworden: Über $90 \%$ besitzen die erforderlichen Rezepte. Die moderne Bildgebung und an der Klinik ausgerichtete experimentelle Modelle haben gezeigt, dass somatische Korrelate auch bei Schmerzformen zu finden sind, die in der Vergangenheit als „histrionisch“ missdeutet wurden. Schmerzen ohne Korrelat waren - zumindenst bei Nervenschmerzen - oft nur falsch untersuchte Schmerzen. Heute verzeichnen wir auch in Deutschland einen bemerkenswerten Anstieg in der Verschreibung nicht nur von Opioiden, sondern auch für das Metamizol, einer in den letzten Jahren zu Unrecht verpönten Substanz. Hinzu kommt, dass sich die Existenz von Ko-Analgetika herumgesprochen hat, zumindest seit mit Gabapentin bzw. Pregabalin verträglichere Alternativen für das Carbamazepin zur Verfügung stehen.

Trotzdem erhalten immer noch bis zu 50\% der Tumorpatienten viel zu spät wirksame Medikamente, und viele Ärzte können mit Begleitsymptomen und unerwünschten Nebenwirkungen schlecht umgehen. Eine aktuelle Initiative mit Titel „Schmerzfreies Krankenhaus“ hat gezeigt, dass auch im Jahre 2004 immer noch bis zur Hälfte aller Patienten in einer Klinik an vermeidbaren, weil sehr starken Schmerzen leiden. Der statistisch entscheidende Risikofaktor für starke Schmerzen ist dabei übrigens nicht etwa die Art der Operation, sondern generell die Klinik. Die Unterschiede in der Schmerzintensität sind zwischen einzelnen Kliniken weitaus größer als innerhalb einer Klinik mit differenten Eingriffen. Darüber hinaus hat diese Untersuchung erstmalig gezeigt, in welchem hohen Umfang auch Patienten in konservativen Abteilungen zum Beispiel mit neurologischen, orthopädischen und internistischen Erkrankungen ebenfalls an Schmerzen leiden.
Dennoch aber ist es aber falsch, stets nur diese Residuen einer Unterversorgung zu beklagen. Seit mehreren Jahren beobachten wir umgekehrt auch das Phänomen einer unkritischen Überversorgung. Böse Zungen sprechen bereits von einer „(Opiat)pflasterseuche“, deren Opfer Patienten mit fragwürdigen Indikationen wie der Fibromyalgie, der somatoformen Schmerzstörung oder generalisierten Schmerzerkrankungen sind. In Schmerzkliniken werden inzwischen immer häufiger Entzugsbehandlungen notwendig, besonders häufig auch nach einer fehlindizierten intrathekalen Opioidgabe.

Das Dilemma, das viele Schmerztherapeuten zum Spagat treibt, ist dieses Nebeneinander von Unter- und scheinbarer Überversorgung, die in Wirklichkeit eine Fehlversorgung ist. Sie gefährdet vieles, was in den letzten Jahrzehnten erreicht wurde. Es ist daher eine wichtige Aufgabe der Schmerztherapeuten selbst, diese Risiken beim Namen zu nennen - auch auf die Gefahr hin, den Beifall von jenen zu bekommen, die selbst bei inkurablen Leiden stets die Symptomlinderung vernachlässigt haben. Praktisch erfordert die Schmerztherapie keinen Spagat, sondern nur die Einhaltung weniger Regeln, wie sich am Beispiel der Opioide demonstrieren lässt. Denn diese sind einfach zu handhaben, wenn Grundregeln beachtet werden: Am wichtigsten ist, dass Patienten Opioide erhalten, wenn sie sie benötigen, also andere Medikamente nicht helfen und die oben genannten Problemkonstellationen nicht bestehen. Die zweitwichtigste Regel lautet: Kontrolliere den Bedarf und den analgetischen Effekt anhand der Angaben des Patienten über eine der vielen hier geeigneten Skalen. Drittens: Setze jedes Analgetikum wieder ab, wenn es nicht hilft.

Je weniger jedoch die Schmerzintensität zu den Befunden passt, je unklarer die Genese ist, je wechselnder die Symptomatik und umso mehr die Vorgeschichte durch Erfolglosigkeit aller Vorbehandlungen gekennzeichnet ist, umso notwendiger sollte die Entscheidung zum Beispiel über eine Opioidtherapie einem interdisziplinär arbeitenden Zentrum überlassen werden. Denn entscheidend ist, dass die Schmerztherapie nicht ein Ziel an sich, sondern „nur“ ein Bestandteil der Gesamtaufgabe ist, Krankheit zu heilen und die Patienten vor schlimmerem Leid zu bewahren.
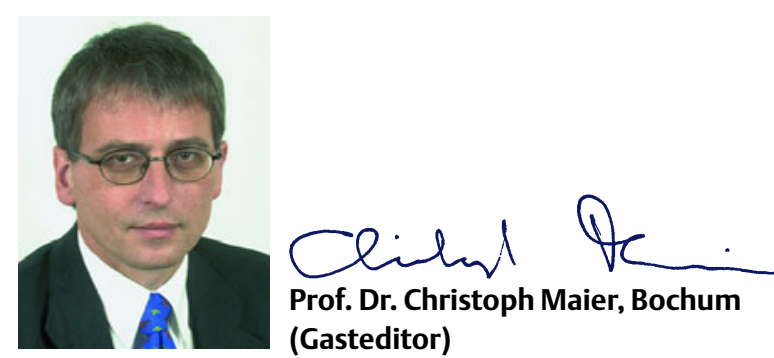
Prof. Dr. Christoph Maier, Bochum (Gasteditor) 